\title{
General decay for viscoelastic plate equation with $p$-Laplacian and time-varying delay
}

\section{Jum-Ran Kang}

"Correspondence:

pointegg@hanmail.net Department of Mathematics,

Dong-A University, Busan, Korea

\begin{abstract}
In this paper we study the viscoelastic plate equation with $p$-Laplacian and time-varying delay. We establish a general decay rate result under some restrictions on the coefficients of strong damping and strong time-varying delay and weakening the usual assumptions on the relaxation function, using the energy perturbation method.
\end{abstract}

MSC: 35B35; 37B25; 74Dxx; 93D20

Keywords: Memory; Plate equation; General decay rate; $p$-Laplacian; Time-varying delay

\section{Introduction}

In this paper, we are concerned with the following problem:

$$
\begin{aligned}
& u_{t t}+\alpha \Delta^{2} u-\Delta_{p} u-\int_{-\infty}^{t} g(t-s) \Delta^{2} u(s) d s-\mu_{1} \Delta u_{t} \\
& -\mu_{2} \Delta u_{t}(t-\tau(t))+f(u)=h, \quad \Omega \times \mathbb{R}^{+}, \\
& u=\Delta u=0 \quad \text { on } \partial \Omega \times \mathbb{R}^{+}, \\
& u(x, 0)=u_{0}(x), \quad u_{t}(x, 0)=u_{1}(x), \quad x \in \Omega, \\
& u_{t}(x, t)=f_{0}(x, t), \quad(x, t) \in \Omega \times[-\tau(0), 0),
\end{aligned}
$$

where $\Omega$ is a bounded domain of $\mathbb{R}^{n}$ with a sufficiently smooth boundary $\partial \Omega$, and

$$
\Delta_{p} u=\operatorname{div}\left(\|\nabla u\|^{p-2} \nabla u\right)
$$

is the $p$-Laplacian operator. The unknown function $u(x, t)$ denotes the transverse displacement of a plate filament with prescribed history $u_{0}(x, t), t \leq 0$. The constants $\alpha$ and $\mu_{1}$ are positive and $\mu_{2}$ is a real number. The function $\tau(t)>0$ represents the time-varying delay, $g>0$ is the memory kernel and $f$ is forcing term.

The plate equation with lower order perturbation of $p$-Laplacian type,

$$
u_{t t}+\Delta^{2} u-\Delta_{p} u-\Delta u_{t}=h(x),
$$

(c) The Author(s) 2018. This article is distributed under the terms of the Creative Commons Attribution 4.0 International License (http://creativecommons.org/licenses/by/4.0/), which permits unrestricted use, distribution, and reproduction in any medium, provided you give appropriate credit to the original author(s) and the source, provide a link to the Creative Commons license, and indicate if changes were made. 
has been extensively studied (see [1-3]) and results concerning existence, nonexistence and long-time behavior of solutions have been considered. This model can be regarded as describing elastoplastic microstructure flows. On the other hand, the elliptic problem for $p$-Laplace operator can be found in [4]. Recently, Torres [5] showed the existence of a solution for the fractional $p$-Laplacian Dirichlet problem with mixed derivatives.

When $\mu_{2}=0$ in Eq. (1.1), that is, in the absence of delay, problem (1.1) with strong damping was investigated by Jorge Silva and Ma [6]. They established exponential stability of solutions under the condition

$$
g^{\prime}(t) \leq-c g(t), \quad \forall t \geq 0,
$$

for some $c>0$. Andrade et al. [7] proved exponential stability of solutions for the plate equation with finite memory and $p$-Laplacian. The viscosity term $-\Delta u_{t}$ is often called a Kelvin-Voigt type dissipation or strong dissipation; it appears in phenomena of wave propagation in a viscoelastic material. Nakao [8] obtained the existence and uniqueness of a global decaying solution for the quasilinear wave equation with Kelvin-Voigt dissipation and a derivative nonlinearity. Pukach et al. [9] studied sufficient conditions of nonexistence of global in time solution for a nonlinear evolution equation with memory generalizing the Voigt-Kelvin model. Recently, Cavalcanti et al. [10] considered intrinsic decay rates for the energy of a nonlinear viscoelastic equation modeling the vibrations of thin rods with variable density.

Time delays so often arise in many physical, chemical, biological, thermal, and economical phenomena because these phenomena depend not only on the present state but also on the past history of the system in a more complicated way. In recent years, there has been published much work concerning the wave equation with constant delay or timevarying delay effects. Nicaise and Pignotti [11] investigated some stability results for the following wave equation with a linear damping and delay term in the domain:

$$
u_{t t}-\Delta u+\mu_{1} u_{t}+\mu_{2} u_{t}(t-\tau)=0
$$

in the case $0<\mu_{2}<\mu_{1}$. Moreover, the same results were obtained when both the damping and the delay act on the boundary. Nicaise and Pignotti [12] studied exponential stability results for the following wave equation with time-dependent delay:

$$
u_{t t}-\Delta u+\mu_{1} u_{t}+\mu_{2} u_{t}(t-\tau(t))=0
$$

under the condition $\left|\mu_{2}\right|<\sqrt{1-d} \mu_{1}$. Kirane and Said-Houari [13] considered the following viscoelastic wave equation with a linear damping and a delay term:

$$
u_{t t}-\Delta u+\int_{0}^{t} g(t-s) \Delta u(s) d s+\mu_{1} u_{t}+\mu_{2} u_{t}(t-\tau)=0,
$$

where $\mu_{1}$ and $\mu_{2}$ are positive constants. When $\mu_{2} \leq \mu_{1}$, they proved general decay of the energy under the condition

$$
g^{\prime}(t) \leq-\xi(t) g(t), \quad \forall t \geq 0,
$$


where $\xi: R^{+} \rightarrow R^{+}$is a nonincreasing differentiable function. Dai and Yang [14] improved the results of [13]. They also obtained an exponentially decay results for the energy of the problem (1.8) in the case $\mu_{1}=0$. Liu [15] studied a general decay result for the following viscoelastic wave equation with time-dependent delay:

$$
u_{t t}-\Delta u+\int_{0}^{t} g(t-s) \Delta u(s) d s+\mu_{1} u_{t}+\mu_{2} u_{t}(t-\tau(t))=0
$$

under the conditions (1.9) and $\left|\mu_{2}\right|<\sqrt{1-d} \mu_{1}$. For the plate equation with time delay term, Yang [16] considered the stability for an Euler-Bernoulli viscoelastic equation with constant delay

$$
u_{t t}+\Delta^{2} u+\int_{0}^{t} g(t-s) \Delta^{2} u(s) d s+\mu_{1} u_{t}+\mu_{2} u_{t}(t-\tau)=0
$$

under the conditions (1.5) and $0<\left|\mu_{2}\right|<\mu_{1}$. Moreover, he proved the exponential decay results of the energy in the case $\mu_{1}=0$. Recently, Feng [17] investigated an exponential stability results for the following plate equation with time-varying delay and past history:

$$
u_{t t}+\alpha \Delta^{2} u-\int_{-\infty}^{t} g(t-s) \Delta^{2} u(s) d s+\mu_{1} u_{t}+\mu_{2} u_{t}(t-\tau(t))+f(u)=0
$$

under the conditions (1.5) and $0<\left|\mu_{2}\right|<\sqrt{1-d} \mu_{1}$. Mustafa and Kafini [18] showed the decay rates for memory type plate system (1.12) with $\tau(t)=\tau$ and $f(u)=-u|u|^{\gamma}$. Park [19] obtained the general decay estimates for a viscoelastic plate equation with time-varying delay under the condition (1.9). The stability of the solutions to a viscoelastic system under the condition (1.9) was studied in [20-23] and the references therein. With respect to wave equation with strong time delay, there is just little published work. Messaoudi et al. [24] considered the following wave equation with strong time delay:

$$
u_{t t}-\Delta u-\mu_{1} \Delta u_{t}-\mu_{2} \Delta u_{t}(t-\tau)=0
$$

and proved the well-posedness under the condition $\left|\mu_{2}\right| \leq \mu_{1}$ and obtained exponential decay of energy under the condition $\left|\mu_{2}\right|<\mu_{1}$. Recently, Feng [25] established the general decay result for the following viscoelastic wave equation with strong time-dependent delay:

$$
u_{t t}-\Delta u+\int_{0}^{t} g(t-s) \Delta u(s) d s-\mu_{1} \Delta u_{t}-\mu_{2} \Delta u_{t}(t-\tau(t))=0
$$

under the conditions (1.9) and $\left|\mu_{2}\right|<\sqrt{1-d} \mu_{1}$.

However, to the best of my knowledge, there is no stability result for the viscoelastic plate equation with strong time-varying delay. Motivated by [24, 25], we study a general decay result for viscoelastic plate equation with $p$-Laplacian and time-varying delay (1.1)-(1.4) for relaxation function $g$ satisfying the condition (1.9). This result improves on earlier ones in the literature because it allows for certain relaxation functions which are not necessarily of exponential or polynomial decay. 
We end this section by establishing the usual history setting of problem (1.1)-(1.4). Following a method devised in [26-29], we shall use a new variable $\eta^{t}$ to the system with past history. Let us define

$$
\eta=\eta^{t}(x, s)=u(x, t)-u(x, t-s), \quad(x, s) \in \Omega \times \mathbb{R}^{+}, t \geq 0 .
$$

Differentiating in (1.15) we have

$$
\eta_{t}^{t}(x, s)+\eta_{s}^{t}(x, s)=u_{t}(x, t), \quad(x, s) \in \Omega \times \mathbb{R}^{+}, t \geq 0 .
$$

Taking $\alpha=1+\int_{0}^{\infty} g(s) d s$, the original problem (1.1)-(1.4) can be transformed into the new system

$$
\left\{\begin{array}{l}
u_{t t}+\Delta^{2} u-\Delta_{p} u+\int_{0}^{\infty} g(s) \Delta^{2} \eta^{t}(s) d s \\
\quad-\mu_{1} \Delta u_{t}-\mu_{2} \Delta u_{t}(t-\tau(t))+f(u)=h, \quad \text { in } \Omega \times \mathbb{R}^{+}, \\
\eta_{t}^{t}=-\eta_{s}^{t}+u_{t}, \quad(x, t, s) \in \Omega \times \mathbb{R}^{+} \times \mathbb{R}^{+}, \\
u_{t}(x, t)=f_{0}(x, t), \quad(x, t) \in \Omega \times[-\tau(0), 0),
\end{array}\right.
$$

with boundary conditions

$$
u(x, t)=0 \quad \text { on } \partial \Omega \times \mathbb{R}^{+}, \quad \eta=0 \quad \text { on } \partial \Omega \times \mathbb{R}^{+} \times \mathbb{R}^{+},
$$

and initial conditions

$$
u(x, 0)=u_{0}(x), \quad u_{t}(x, 0)=u_{1}(x), \quad \eta^{t}(x, 0)=0, \quad \eta^{0}(x, s)=\eta_{0}(x, s),
$$

where

$$
\left\{\begin{array}{l}
u_{0}(x)=u_{0}(x, 0), \quad x \in \Omega, \\
u_{1}(x)=\left.\partial_{t} u_{0}(x, t)\right|_{t=0}, \quad x \in \Omega, \\
\eta_{0}(x, s)=u_{0}(x, 0)-u_{0}(x,-s), \quad(x, s) \in \Omega \times \mathbb{R}^{+} .
\end{array}\right.
$$

The paper is organized as follows. In Sect. 2, we state the notation and main result. In Sect. 3, we prove the general decay of the solutions to the viscoelastic plate equation with $p$-Laplacian and time-varying delay by using the energy perturbation method.

\section{Preliminaries}

In this section, we present some material needed in the proof of our result and state the main result.

For a Banach space $X,\|\cdot\|_{X}$ denotes the norm of $X$. For simplicity, we denote $\|\cdot\|_{L^{2}(\Omega)}$ by $\|\cdot\|$. We use the standard Lebesgue and Sobolev spaces, with their usual scalar products and norms, and the Sobolev-Poincaré inequality

$$
\|u\|_{q}^{2} \leq C_{*}\|\Delta u\|^{2}, \quad u \in H_{0}^{2}(\Omega)
$$

for $q \geq 2$ if $1 \leq n \leq 4$ or $2 \leq q \leq \frac{2 n}{n-4}$ if $n \geq 5$. 
In the following, we fix some notations on the function spaces that will be used. Let

$$
V_{0}=L^{2}(\Omega), \quad V_{1}=H_{0}^{1}(\Omega), \quad V_{2}=H^{2}(\Omega) \cap H_{0}^{1}(\Omega)
$$

and

$$
V_{3}=\left\{u \in H^{3}(\Omega) \mid u=\Delta u=0 \text { on } \partial \Omega\right\} .
$$

In order to consider the new variable $\eta$, we introduce the weighted $L^{2}$-spaces

$$
\mathcal{M}_{i}=L_{g}^{2}\left(\mathbb{R}^{+} ; V_{i}\right)=\left\{\eta: \mathbb{R}^{+} \rightarrow V_{i} \mid \int_{0}^{\infty} g(s)\|\eta(s)\|_{V_{i}}^{2} d s<\infty\right\}, \quad i=0,1,2,3,
$$

which are Hilbert spaces endowed with the inner products and norms

$$
(\eta, \xi)_{\mathcal{M}_{i}}=\int_{0}^{\infty} g(s)(\eta(s), \xi(s))_{V_{i}} d s \quad \text { and } \quad\|\eta\|_{\mathcal{M}_{i}}^{2}=\int_{0}^{\infty} g(s)\|\eta(s)\|_{V_{i}}^{2} d s, \quad i=0,1,2,3,
$$

respectively. To simplify the notation, we define the Hilbert spaces

$$
\mathcal{H}=V_{2} \times V_{0} \times \mathcal{M}_{2} \text { and } \mathcal{H}_{1}=V_{3} \times V_{1} \times \mathcal{M}_{3}
$$

Let us begin with the precise hypotheses on the constant $p$ and the functions $f$ and $g$. For $n \in \mathbb{N}$, we assume that

$$
2 \leq p \leq \frac{2 n-2}{n-2} \quad \text { if } n \geq 3 \quad \text { and } \quad p \geq 2 \quad \text { if } n=1,2 .
$$

Then

$$
H^{2}(\Omega) \cap H_{0}^{1}(\Omega) \hookrightarrow W_{0}^{1,2(p-1)}(\Omega) \hookrightarrow H_{0}^{1}(\Omega) \hookrightarrow L^{2}(\Omega)
$$

The nonlinear function $f: \mathbb{R} \rightarrow \mathbb{R}$ satisfying $f(0)=0$ and the growth condition,

$$
|f(w)-f(z)| \leq k_{0}\left(1+|w|^{\rho}+|z|^{\rho}\right)|w-z|, \quad \forall w, z \in \mathbb{R},
$$

where $k_{0}>0$ and

$$
0<\rho \leq \frac{4}{n-4} \quad \text { if } n \geq 5 \quad \text { and } \quad \rho>0 \quad \text { if } 1 \leq n \leq 4,
$$

which implies that $H^{2}(\Omega) \hookrightarrow L^{2(\rho+1)}(\Omega)$. In addition, we assume that

$$
0 \leq F(u) \leq f(u) u, \quad \forall u \in \mathbb{R},
$$

where $F(z)=\int_{0}^{z} f(s) d s$.

For the relaxation function $g$, we assume that $g: \mathbb{R}^{+} \rightarrow \mathbb{R}^{+}$is a nonincreasing $C^{1}$ function satisfying

$$
g(0)>0, \quad l:=1-\int_{0}^{\infty} g(s) d s>0,
$$


and there exists a nonincreasing differentiable function $\xi: \mathbb{R}^{+} \rightarrow \mathbb{R}^{+}$satisfying

$$
\xi(t)>0, g^{\prime}(t) \leq-\xi(t) g(t), \quad \forall t \geq 0
$$

and

$$
\int_{0}^{+\infty} \xi(t) d t=\infty
$$

As in [12], for the time-varying delay, we assume that $\tau \in W^{2, \infty}([0, T])$ for $T>0$, and there exist positive constants $\tau_{0}, \tau_{1}$ and $d$ satisfying

$$
0<\tau_{0} \leq \tau(t) \leq \tau_{1} \quad \text { and } \quad \tau^{\prime}(t) \leq d<1 \quad \forall t>0
$$

and that $\mu_{1}$ and $\mu_{2}$ satisfy

$$
\left|\mu_{2}\right|<\sqrt{1-d} \mu_{1}
$$

We can prove the existence of weak solution by making use of the classical FaedoGalerkin method. Then using elliptic regularity and second order estimates we can show the regularity of the solution. We state a well-posedness result without a proof here (see $[6,7,13,17])$.

Theorem 2.1 Suppose that hypotheses (2.2)-(2.9) hold.

(i) If the initial data $\left(u_{0}, u_{1}, \eta_{0}\right) \in \mathcal{H}, f_{0}(x, t) \in H^{1}(\Omega \times(-\tau(0), 0))$ and $h(x) \in L^{2}(\Omega)$, then problem (1.16)-(1.18) has a unique weak solution

$$
\left(u, u_{t}, \eta^{t}\right) \in C(0, T ; \mathcal{H}), \quad \forall T>0,
$$

satisfying

$$
u \in L^{\infty}\left(0, T ; V_{2}\right), \quad u_{t} \in L^{\infty}\left(0, T ; V_{0}\right) \cap L^{2}\left(0, T ; V_{1}\right), \quad \eta^{t} \in L^{\infty}\left(0, T ; \mathcal{M}_{2}\right) .
$$

(ii) If the initial data $\left(u_{0}, u_{1}, \eta_{0}\right) \in \mathcal{H}_{1}, f_{0}(x, t) \in H^{2}(\Omega \times(-\tau(0), 0))$ and $h(x) \in H^{1}(\Omega)$, then the above weak solution has higher regularity

$$
\begin{aligned}
& u \in L^{\infty}\left(0, T ; V_{3}\right), \quad u_{t} \in L^{\infty}\left(0, T ; V_{1}\right) \cap L^{2}\left(0, T ; V_{2}\right), \\
& \eta^{t} \in L^{\infty}\left(0, T ; \mathcal{M}_{3}\right) .
\end{aligned}
$$

In order to state our main result, we define the energy of problem (1.16)-(1.18) by

$$
\begin{aligned}
E(t)= & \frac{1}{2}\left\|u_{t}(t)\right\|^{2}+\frac{1}{2}\|\Delta u(t)\|^{2}+\frac{1}{p}\|\nabla u(t)\|_{p}^{p}+\int_{\Omega} F(u) d x \\
& +\frac{1}{2}\left\|\eta^{t}\right\|_{\mathcal{M}_{2}}^{2}+\frac{\xi}{2} \int_{t-\tau(t)}^{t} e^{\lambda(s-t)}\left\|\nabla u_{t}(s)\right\|^{2} d s,
\end{aligned}
$$


where $\xi$ and $\lambda$ are positive constants satisfying

$$
\frac{\left|\mu_{2}\right|}{\sqrt{1-d}}<\xi<2 \mu_{1}-\frac{\left|\mu_{2}\right|}{\sqrt{1-d}} \text { and } \lambda<\frac{1}{\tau_{1}} \log \left|\frac{\xi \sqrt{1-d}}{\left|\mu_{2}\right|}\right| .
$$

Note that this choice of $\xi$ is possible from assumption (2.9).

Theorem 2.2 Suppose that assumptions (2.2)-(2.9) hold. Let $h=0$ and the initial data $\left(u_{0}, u_{1}, \eta_{0}\right) \in \mathcal{H}, f_{0}(x, t) \in H^{1}(\Omega \times(-\tau(0), 0))$. Then there exist two positive constants $k_{1}$ and $k_{2}$ such that the energy $E(t)$ satisfies

$$
E(t) \leq k_{1} e^{-k_{2} \int_{0}^{t} \xi(s) d s}, \quad \forall t \geq 0 .
$$

\section{General decay of the energy}

In this section we shall establish the decay rates in Theorem 2.2. To demonstrate the stability of the system (1.16)-(1.18) the lemmas below are essential.

Lemma 3.1 Under the assumptions of Theorem 2.2, the energy functional $E(t)$ satisfies, for any $t \geq 0$,

$$
\begin{aligned}
E^{\prime}(t) \leq & \left(\frac{\left|\mu_{2}\right|}{2 \sqrt{1-d}}-\mu_{1}+\frac{\xi}{2}\right)\left\|\nabla u_{t}\right\|^{2}+\left(\frac{\left|\mu_{2}\right| \sqrt{1-d}}{2}-\frac{\xi(1-d)}{2 e^{\lambda \tau_{1}}}\right)\left\|\nabla u_{t}(t-\tau(t))\right\|^{2} \\
& +\frac{1}{2} \int_{0}^{\infty} g^{\prime}(s)\left\|\Delta \eta^{t}(s)\right\|^{2} d s-\frac{\lambda \xi}{2} \int_{t-\tau(t)}^{t} e^{\lambda(s-t)}\left\|\nabla u_{t}(s)\right\|^{2} d s
\end{aligned}
$$

Proof Multiplying the first equation of $(1.16)$ by $u_{t}(t)$, we get the identity

$$
\begin{aligned}
& \frac{d}{d t}\left(\frac{1}{2}\left\|u_{t}\right\|^{2}+\frac{1}{2}\|\Delta u\|^{2}+\frac{1}{p}\|\nabla u\|_{p}^{p}+\int_{\Omega} F(u) d x\right) \\
& \quad=-\mu_{1}\left\|\nabla u_{t}\right\|^{2}-\mu_{2} \int_{\Omega} \nabla u_{t}(t-\tau(t)) \nabla u_{t} d x-\int_{\Omega} \int_{0}^{\infty} g(s) \Delta \eta^{t}(s) \Delta u_{t}(t) d s d x
\end{aligned}
$$

Applying the second equation of (1.16) to (3.2), we have

$$
\begin{aligned}
E^{\prime}(t)= & -\mu_{1}\left\|\nabla u_{t}\right\|^{2}-\mu_{2} \int_{\Omega} \nabla u_{t}(t-\tau(t)) \nabla u_{t} d x-\left(\eta_{s}^{t}, \eta^{t}\right)_{\mathcal{M}_{2}}+\frac{\xi}{2}\left\|\nabla u_{t}\right\|^{2} \\
& -\frac{\xi}{2} e^{-\lambda \tau(t)}\left(1-\tau^{\prime}(t)\right)\left\|\nabla u_{t}(t-\tau(t))\right\|^{2}-\frac{\lambda \xi}{2} \int_{t-\tau(t)}^{t} e^{\lambda(s-t)}\left\|\nabla u_{t}(s)\right\|^{2} d s .
\end{aligned}
$$

Using Young's inequality, we obtain

$$
-\mu_{2} \int_{\Omega} \nabla u_{t}(t-\tau(t)) \nabla u_{t} d x \leq \frac{\left|\mu_{2}\right|}{2 \sqrt{1-d}}\left\|\nabla u_{t}\right\|^{2}+\frac{\left|\mu_{2}\right| \sqrt{1-d}}{2}\left\|\nabla u_{t}(t-\tau(t))\right\|^{2}
$$

By (2.8) we get

$$
-\frac{\xi}{2} e^{-\lambda \tau(t)}\left(1-\tau^{\prime}(t)\right)\left\|\nabla u_{t}(t-\tau(t))\right\|^{2} \leq-\frac{\xi}{2} e^{-\lambda \tau_{1}}(1-d)\left\|\nabla u_{t}(t-\tau(t))\right\|^{2} .
$$


Since $\eta^{t}(0)=0$, we have

$$
-\left(\eta_{s}^{t}, \eta^{t}\right)_{\mathcal{M}_{2}}=\frac{1}{2} \int_{0}^{\infty} g^{\prime}(s)\left\|\Delta \eta^{t}(s)\right\|^{2} d s .
$$

Combining with the above estimates, we obtain the desired inequality (3.1). The proof is now complete.

Now, let us define the perturbed modified energy by

$$
L(t)=E(t)+\epsilon \Phi(t),
$$

where

$$
\Phi(t)=\int_{\Omega} u_{t} u d x
$$

Then it is easily shown that there exists $C_{1}>0$ such that

$$
|L(t)-E(t)| \leq \epsilon C_{1} E(t), \quad \forall t \geq 0, \forall \epsilon>0,
$$

where $C_{1}=\max \left\{1, C_{*}\right\}$.

Lemma 3.2 There exist positive constants $C_{2}$ and $C_{3}$ such that

$$
L^{\prime}(t) \leq-C_{2} E(t)+C_{3} \int_{0}^{\infty} g(s)\left\|\Delta \eta^{t}(s)\right\|^{2} d s, \quad \forall t \geq 0 .
$$

Proof By using (1.16), we get

$$
\begin{aligned}
\Phi^{\prime}(t)= & \left\|u_{t}\right\|^{2}-\|\Delta u\|^{2}-\|\nabla u\|_{p}^{p}-\int_{\Omega} \int_{0}^{\infty} g(s) \Delta \eta^{t}(s) \Delta u(t) d s d x \\
& -\mu_{1} \int_{\Omega} \nabla u_{t} \nabla u d x-\mu_{2} \int_{\Omega} \nabla u_{t}(t-\tau(t)) \nabla u d x-\int_{\Omega} f(u) u d x .
\end{aligned}
$$

Adding and subtracting $E(t)$, we see that

$$
\begin{aligned}
\Phi^{\prime}(t)= & -E(t)+\frac{3}{2}\left\|u_{t}\right\|^{2}-\frac{1}{2}\|\Delta u\|^{2}-\left(1-\frac{1}{p}\right)\|\nabla u\|_{p}^{p}+\frac{1}{2}\left\|\eta^{t}\right\|_{\mathcal{M}_{2}}^{2} \\
& +\int_{\Omega}(F(u)-f(u) u) d x \\
& -\int_{\Omega} \int_{0}^{\infty} g(s) \Delta \eta^{t}(s) \Delta u(t) d s d x-\mu_{1} \int_{\Omega} \nabla u_{t} \nabla u d x \\
& -\mu_{2} \int_{\Omega} \nabla u_{t}(t-\tau(t)) \nabla u d x \\
& +\frac{\xi}{2} \int_{t-\tau(t)}^{t} e^{\lambda(s-t)}\left\|\nabla u_{t}(s)\right\|^{2} d s .
\end{aligned}
$$


Applying Young's inequality and (2.6), we have

$$
\begin{aligned}
& \int_{\Omega} \int_{0}^{\infty} g(s) \Delta \eta^{t}(s) \Delta u(t) d s d x \leq \frac{1}{8}\|\Delta u\|^{2}+2(1-l)\left\|\eta^{t}\right\|_{\mathcal{M}_{2}}^{2}, \\
& -\mu_{1} \int_{\Omega} \nabla u_{t} \nabla u d x \leq \frac{1}{16}\|\Delta u\|^{2}+4 d_{1} \mu_{1}^{2}\left\|\nabla u_{t}\right\|^{2}
\end{aligned}
$$

and

$$
-\mu_{2} \int_{\Omega} \nabla u_{t}(t-\tau(t)) \nabla u d x \leq \frac{1}{16}\|\Delta u\|^{2}+4 d_{1} \mu_{2}^{2}\left\|\nabla u_{t}(t-\tau(t))\right\|^{2},
$$

where $d_{1}>0$ is an embedding constant for $H^{2}(\Omega) \cap H_{0}^{1}(\Omega) \hookrightarrow H_{0}^{1}(\Omega)$. Combining all above estimates and using (2.5), we obtain

$$
\begin{aligned}
\Phi^{\prime}(t) \leq & -E(t)+\left(4 d_{1} \mu_{1}^{2}+\frac{3}{2} d_{2}\right)\left\|\nabla u_{t}\right\|^{2}-\frac{1}{4}\|\Delta u\|^{2} \\
& -\left(1-\frac{1}{p}\right)\|\nabla u\|_{p}^{p}+\left(2(1-l)+\frac{1}{2}\right)\left\|\eta^{t}\right\|_{\mathcal{M}_{2}}^{2} \\
& +4 d_{1} \mu_{2}^{2}\left\|\nabla u_{t}(t-\tau(t))\right\|^{2}+\frac{\xi}{2} \int_{t-\tau(t)}^{t} e^{\lambda(s-t)}\left\|\nabla u_{t}(s)\right\|^{2} d s,
\end{aligned}
$$

where $d_{2}>0$ is an embedding constant for $H_{0}^{1}(\Omega) \hookrightarrow L^{2}(\Omega)$. Thus, taking two positive constants

$$
c_{1}=\max \left\{4 d_{1} \mu_{1}^{2}+\frac{3}{2} d_{2}, 4 d_{1} \mu_{2}^{2}\right\} \quad \text { and } \quad c_{2}=2(1-l)+\frac{1}{2}
$$

we find that

$$
\begin{aligned}
\Phi^{\prime}(t) \leq & -E(t)+c_{1}\left\|\nabla u_{t}\right\|^{2}+c_{1}\left\|\nabla u_{t}(t-\tau(t))\right\|^{2} \\
& +c_{2}\left\|\eta^{t}\right\|_{\mathcal{M}_{2}}^{2}+\frac{\xi}{2} \int_{t-\tau(t)}^{t} e^{\lambda(s-t)}\left\|\nabla u_{t}(s)\right\|^{2} d s .
\end{aligned}
$$

Using (3.1), (3.3) and (3.5), we deduce that

$$
\begin{aligned}
L^{\prime}(t) \leq & -\epsilon E(t)+\left(\frac{\left|\mu_{2}\right|}{2 \sqrt{1-d}}-\mu_{1}+\frac{\xi}{2}+\epsilon c_{1}\right)\left\|\nabla u_{t}\right\|^{2} \\
& +\left(\frac{\left|\mu_{2}\right| \sqrt{1-d}}{2}-\frac{\xi(1-d)}{2 e^{\lambda \tau_{1}}}+\epsilon c_{1}\right)\left\|\nabla u_{t}(t-\tau(t))\right\|^{2} \\
& +\frac{1}{2} \int_{0}^{\infty} g^{\prime}(s)\left\|\Delta \eta^{t}(s)\right\|^{2} d s+\epsilon c_{2}\left\|\eta^{t}\right\|_{\mathcal{M}_{2}}^{2}-\frac{\xi}{2}(\lambda-\epsilon) \int_{t-\tau(t)}^{t} e^{\lambda(s-t)}\left\|\nabla u_{t}(s)\right\|^{2} d s .
\end{aligned}
$$

From (2.10), we see that

$$
\frac{\left|\mu_{2}\right|}{2 \sqrt{1-d}}-\mu_{1}+\frac{\xi}{2}<0, \quad \frac{\left|\mu_{2}\right| \sqrt{1-d}}{2}-\frac{\xi(1-d)}{2 e^{\lambda \tau_{1}}}<0 .
$$


We choose $\epsilon>0$ sufficiently small for

$$
\frac{\left|\mu_{2}\right|}{2 \sqrt{1-d}}-\mu_{1}+\frac{\xi}{2}+\epsilon c_{1}<0, \quad \frac{\left|\mu_{2}\right| \sqrt{1-d}}{2}-\frac{\xi(1-d)}{2 e^{\lambda \tau_{1}}}+\epsilon c_{1}<0 \quad \text { and } \quad \lambda-\epsilon>0 .
$$

Hence, we conclude that (3.4) holds for some constants $C_{2}, C_{3}>0$.

Proof of Theorem 2.2 From the ideas presented in [19-23], we get the following results. Multiplying (3.4) by $\xi(t)$ and using (2.7) and (3.1), we get

$$
\begin{aligned}
\xi(t) L^{\prime}(t) & \leq-C_{2} \xi(t) E(t)+C_{3} \lim _{t \rightarrow \infty} \int_{0}^{t} \xi(s) g(s)\left\|\Delta \eta^{t}(s)\right\|^{2} d s \\
& \leq-C_{2} \xi(t) E(t)-C_{3} \int_{0}^{\infty} g^{\prime}(s)\left\|\Delta \eta^{t}(s)\right\|^{2} d s \\
& \leq-C_{2} \xi(t) E(t)-2 C_{3} E^{\prime}(t) .
\end{aligned}
$$

Using the fact that $\xi^{\prime}(t) \leq 0$ and letting

$$
\mathcal{L}(t)=\xi(t) L(t)+2 C_{3} E(t) \sim E(t)
$$

we have

$$
\mathcal{L}^{\prime}(t) \leq-C_{2} \xi(t) E(t) \leq-C_{4} \xi(t) \mathcal{L}(t), \quad \forall t \geq 0,
$$

where $C_{4}$ is a positive constant. A simple integrating of $(3.7)$ over $(0, t)$ leads to

$$
\mathcal{L}(t) \leq \mathcal{L}(0) e^{-C_{4} \int_{0}^{t} \xi(s) d s}, \quad \forall t \geq 0 .
$$

Consequently, from (3.6) and (3.8), we obtain the desired inequality (2.11).

\section{Conclusions}

In this paper, a viscoelastic plate equation with $p$-Laplacian and time-varying delay has been investigated. In recent years, there has been published much work concerning the wave equation with constant delay or time-varying delay. However, to the best of my knowledge, there was no stability result for the viscoelastic plate equation with strong time delay. We have been proved that the general decay rate result under some restrictions on the coefficients of strong damping and strong time-varying delay. Furthermore, we have been obtained the general decay rate result under weakening the usual assumptions on the relaxation function, using the energy perturbation method. This result improves on earlier ones in the literature because it allows for certain relaxation functions which are not necessarily of exponential or polynomial decay. 
Authors' contributions

The work was realized by the author. The author read and approved the final manuscript.

\section{Publisher's Note}

Springer Nature remains neutral with regard to jurisdictional claims in published maps and institutional affiliations.

\section{Received: 7 December 2017 Accepted: 9 February 2018 Published online: 02 April 2018}

\section{References}

1. Yang, Z:: Longtime behavior for a nonlinear wave equation arising in elasto-plastic flow. Math. Methods Appl. Sci. 32, 1082-1104 (2009)

2. Yang, Z:: Global attractors and their Hausdorff dimensions for a class of Kirchhoff models. J. Math. Phys. 51, 032701 (2010)

3. Yang, Z., Jin, B.: Global attractor for a class of Kirchhoff models. J. Math. Phys. 50, 032701 (2009)

4. Ghergu, M., Radulescu, V.: Nonlinear PDEs. Mathematical Models in Biology, Chemistry and Population Genetics. Springer Monographs in Mathematics. Springer, Heidelberg (2012)

5. Torres, C.: Boundary value problem with fractional $p$-Laplacian operator. Adv. Nonlinear Anal. 5(2), 133-146 (2016)

6. Jorge Silva, M.A., Ma, T.F.: On a viscoelastic plate equation with history setting and perturbation of $p$-Laplacian type. IMA J. Appl. Math. 78, 1130-1146 (2013)

7. Andrade, D., Jorge Silva, M.A., Ma, T.F.,: Exponential stability for a plate equation with $p$-Laplacian and memory terms. Math. Methods Appl. Sci. 35, 417-426 (2012)

8. Nakao, M.: Global solutions to the initial-boundary value problem for the quasilinear viscoelastic equation with a derivative nonlinearity. Opusc. Math. 34(3), 569-590 (2014)

9. Pukach, P., I''kiv, V., Nytrebych, Z., Vovk, M.: On nonexistence of global in time solution for a mixed problem for a nonlinear evolution equation with memory generalizing the Voigt-Kelvin rheological model. Opusc. Math. 37(5), 735-753 (2017)

10. Cavalcanti, M.M., Domingos Cavalcanti, V.N., Lasiecka, I., Webler, C.M.: Intrinsic decay rates for the energy of a nonlinear viscoelastic equation modeling the vibrations of thin rods with variable density. Adv. Nonlinear Anal. 6(2), 121-145 (2017)

11. Nicaise, S., Pignotti, C.: Stability and instability results of the wave equation with a delay term in the boundary or internal feedbacks. SIAM J. Control Optim. 45(5), 1561-1585 (2006)

12. Nicaise, S., Pignotti, C.: Interior feedback stabilization of wave equations with time dependence delay. Electron. J. Differ. Equ. 2011, 41 (2011)

13. Kirane, M., Said-Houari, B.: Existence and asymptotic stability of a viscoelastic wave equation with a delay. Z. Angew. Math. Phys. 62, 1065-1082 (2011)

14. Dai, Q., Yang, Z.F.: Global existence and exponential decay of the solution for a viscoelastic wave equation with a delay. Z. Angew. Math. Phys. 65, 885-903 (2014)

15. Liu, W.J.: General decay of the solution for a viscoelastic wave equation with a time-varying delay term in the internal feedback. J. Math. Phys. 54, 043504 (2013)

16. Yang, Z.F.: Existence and energy decay of solutions for the Euler-Bernoulli viscoelastic equation with a delay. Z. Angew. Math. Phys. 66, 727-745 (2015)

17. Feng, B.: Well-posedness and exponential stability for a plate equation with time-varying delay and past history Z. Angew. Math. Phys. 68(6), 1-24 (2017)

18. Mustafa, M.I., Kafini, M.: Decay rates for memory-type plate system with delay and source term. Math. Methods Appl. Sci. 40, 883-895 (2017)

19. Park, S.H.: Decay rate estimates for a weak viscoelastic beam equation with time-varying delay. Appl. Math. Lett. 31 46-51 (2014)

20. Ferreira, J., Messaoudi, S.A.: On the general decay of a nonlinear viscoelastic plate equation with a strong damping and $\vec{p}(x, t)$-Laplacian. Nonlinear Anal. 104, 40-49 (2014)

21. Messaoudi, S.A.: General decay of the solution energy in a viscoelastic equation with a nonlinear source. Nonlinear Anal. 69, 2589-2598 (2008)

22. Messaoudi, S.A., Mustafa, M.l.: On convexity for energy decay rates of a viscoelastic equation with boundary feedback. Nonlinear Anal. 72, 3602-3611 (2010)

23. Park, J.Y., Park, S.H.: Decay rate estimates for wave equations of memory type with acoustic boundary conditions. Nonlinear Anal. 74, 993-998 (2011)

24. Messaoudi, S.A., Fareh, A., Doudi, N.: Well posedness and exponential stability in a wave equation with a strong damping and a strong delay. J. Math. Phys. 57, 111501 (2016)

25. Feng, B.: General decay for a viscoelastic wave equation with strong time-dependent delay. Bound. Value Probl. 2017, 57 (2017)

26. Dafermos, C.M.: Asymptotic stability in viscoelasticity. Arch. Ration. Mech. Anal. 37, 297-308 (1970)

27. Fabrizio, M., Giorgi, C., Pata, V.: A new approach to equations with memory. Arch. Ration. Mech. Anal. 198, 189-232 (2010)

28. Giorgi, C., Rivera, J.E.M., Pata, V.: Global attractors for a semilinear hyperbolic equation in viscoelasticity. J. Math. Anal. Appl. 260, 83-99 (2001)

29. Pata, V., Zucchi, A.: Attractors for a damped hyperbolic equation with linear memory. Adv. Math. Sci. Appl. 11, 505-529 (2001) 